\title{
Cleft lip and palate, sensorineural deafness, and sacral lipoma in two brothers: a possible example of the disorganisation mutant
}

\author{
R Brian Lowry, Siu-Li Yong
}

\begin{abstract}
We report two brothers of Chinese origin who have an apparently unique syndrome of cleft lip/palate, profound sensorineural deafness, and a sacral lipoma. Additional findings which were not common to both were aberrant digital appendages on the heel and thigh of one boy and an anterior sacral meningocele and dislocated hip in the other. Intelligence is normal in both. Both boys suffer from functional constipation but biopsy studies showed no evidence of Hirschsprung's disease. The parents, who are normal, are not related. Inheritance is probably autosomal or $X$ linked recessive. A possible link with the disorganisation mouse mutant is discussed.
\end{abstract}

There are more than 150 syndromes in which cleft lip \pm palate or cleft palate are component features ${ }^{1}$ and since this review by Cohen many additional ones have been described. Most are Mendelian disorders with only a small number having a chromosomal or teratogenic aetiology. Here we report two brothers of Chinese origin with an apparently unique combination of anomalies.

\section{Case reports}

CASE 1

The proband who was born in 1974 was the second child of a healthy, unaffected, unrelated Chinese

Division of Medical Genetics, Department of Paediatrics, University of Calgary and Alberta Children's Hospital Research Centre, 1820 Richmond Road SW, Calgary, Alberta, Canada T2T 5C7.

R B Lowry

Department of Medical Genetics, University of British Columbia, University Hospital, Shaughnessy Site, Vancouver, BC, Canada.

S-L Yong

Correspondence to Dr Lowry.

Received for publication 9 August 1990

Accepted for publication 7 September 1990. couple. At the time of birth, his father was 27 and his mother 25 years. His older brother is normal. The mother had one previous spontaneous abortion. The pregnancy and delivery (birth weight $2860 \mathrm{~g}$, gestation 41 weeks) were unremarkable. The principal clinical features are listed in the table. His facial cleft was extreme with virtually no premaxilla, thus simulating a median cleft lip; however, the nasal septum was attached to the right maxillary process. Thus the classification of his cleft is more accurately a severe left sided cleft lip with a complete cleft palate. Nasal bone and cartilage were poorly developed resulting in a very flat facial profile. On his scalp there was a small, midline, circular area with no hair, having the appearance of a scar, that is, cutis aplasia congenita. His right foot had an extra digit which was attached to the lateral side of his heel. This was a firm structure and contained two bony structures similar to phalanges. There was a second digit-like appendage attached to the lateral aspect of the right thigh (figure). This was soft, did not appear to contain bone, and had two constriction rings. It was attached by a very fine strand of tissue and was removed at birth. The heel appendage was removed at 3 months at the same time as his lip repair. He subsequently had a palate repair at 18 months, ear cartilage transplanted to his nose at 6 years, and further facial reconstruction procedures including an iliac bone graft to the maxillary alveolus. He also had removal of an accessory calcaneus from the lateral aspect of the right heel.

Principal clinical features.

\begin{tabular}{lcc}
\hline & Case 1 & Case 2 \\
\hline Cleft lip and palate & + & + \\
Profound sensorineural deafness & + & + \\
Sacral lipoma & + & + \\
Lower limb asymmetry & + & + \\
Functional constipation & + & + \\
Aberrant polydactyly & + & - \\
Exotropia & + & - \\
Cutis aplasia of scalp & + & + \\
Dislocated hip & - & + \\
Anterior sacral meningocele & - & + \\
Intelligence normal & + & + \\
Growth normal & + & + \\
Rotation defect of penis & - & + \\
\hline
\end{tabular}




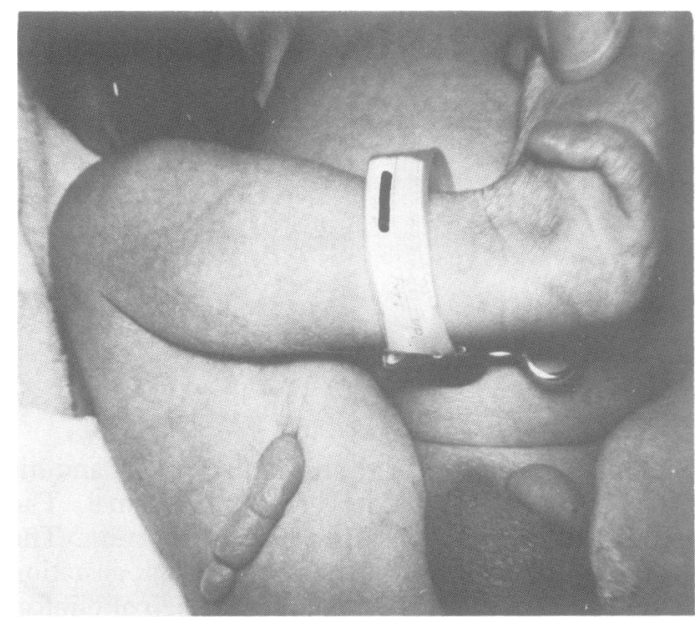

Right leg of case 1 with digit-like structures on thigh and heel.

Hearing loss was suspected by the mother at 3 months but not confirmed until 18 months. His motor development was slow; he sat at 18 months and walked at $2 \frac{1}{2}$ years. His coordination was not good and he was described as being hyperactive with a short attention span, but this has improved with age. Formal testing indicates that he is of normal intelligence. $\mathrm{G}$ banded karyotype was normal $(46, \mathrm{XY})$. A rectal examination was normal except for the presence of hard stool. Because of persistent constipation a barium enema was done which showed mild tapering of the rectum. The rectal ampulla was distensible but the calibre was slightly less than the distal sigmoid. A rectal biopsy was normal with abundant ganglia and ganglion cells in the submucosal and myenteric plexuses. $\mathrm{He}$ and his brother attend a school for the deaf and communicate by signing. There is no oral communication. He still has constipation with very hard stools. There are no urinary problems.

Re-examination at the age of 15 years 4 months showed normal growth (height $164 \mathrm{~cm}$ (25th centile), arm span $163.5 \mathrm{~cm}$, weight $58.5 \mathrm{~kg}$ (50th centile), - OFC $56 \mathrm{~cm}$ (60th centile), inner canthal distance $3 \cdot 2$ $\mathrm{cm}$, interpupillary $6.4 \mathrm{~cm}$, outer canthal $9 \mathrm{~cm}$ (all approximately 50th centile)). He has facial asymmetry with the left cheek being fuller than the right. His neck is tilted to the right but there is no true torticollis as there is a full range of movement. The posterior hairline is not low and there is no neck webbing. A repaired left cleft lip and repaired palate are evident. His maxillary teeth show irregularity with missing upper left central and lateral incisors. His ears are normal in shape, size, and position, there are no simian creases nor clinodactyly, and his dermatoglyphic patterns are unremarkable, with proximally placed axial triradii. The right thigh is larger than the left and there is a diffuse palpable mass in the right buttock which obliterates the gluteal fold and has palpable characteristics of a lipoma. His CNS examination is normal apart from his deafness. Reflexes are symmetrical and tone and power are normal. The anal sphincter tone is normal and there is no demonstrable perianal or sacral sensory loss. The remainder of the physical examination was normal and there is no evidence of a spinal defect.

CASE 2

He was born three years later and had an essentially similar phenotype with a severe left cleft lip and complete cleft palate. He was born prematurely (36 weeks' gestation) and had a birth weight of $2350 \mathrm{~g}$. He also has a flat facial profile and profound senorineural deafness. He did not have any aberrant digital appendages nor cutis aplasia, but he did have a right congenital dislocated hip and functional constipation. A barium enema and rectal biopsy disclosed similar findings to those described in his brother. He also had a sacral lipoma and an anterior sacral meningocele.

The sacral mass was not evident at birth; however, it grew so rapidly that it was thought to be a cystic sacral teratoma. This proved not to be the case as histologically it was a lipoma which extended down into the dura. This was traced to an anterior presacral meningocele. Postoperatively his anal sphincter was patulous but subsequently became normal. This has been confirmed in several subsequent examinations as has normal perianal sensation. Despite this he still has functional constipation with overflow.

Motor development was normal (sat at 8 months, walked at 15 months). His hearing loss was diagnosed much earlier and total communication started at 1 year. His intelligence is normal. At the age of 12 years 6 months he was re-examined. Height was $149.3 \mathrm{~cm}$ ( 25 th to 50 th centile), arm span $148.7 \mathrm{~cm}$, OFC 54.3 $\mathrm{cm}$ (25th to 50th centile), inner canthal, interpupillary, and outer canthal measurements $3 \cdot 1,5 \cdot 5$, and $8 \cdot 2 \mathrm{~cm}$ respectively (all approximately 50 th centile). Physical examination disclosed no abnormalities except for his repaired left cleft lip, repaired cleft palate, very mild lower limb asymmetry with the left being slightly longer $(0.8 \mathrm{~cm})$, and a rotational deformity of the penis indicating hypoplasia or aplasia of one of the corpora cavernosa. His buttocks are an abnormal shape owing to previous surgery which had been complicated by postoperative skin necrosis resulting in considerable scarring. He has a full set of normal teeth apart from irregularities in eruption and the shape of his maxillary arch. There are no simian creases and his dermatoglyphics are unremarkable. His deep tendon reflexes were difficult to obtain but were symmetrical with downward plantar reflexes. 


\section{Discussion}

We believe that the sibs we have described fall into a new clefting syndrome category. The key features are severe cleft lip and palate, profound sensorineural hearing loss, and a lipoma of the buttock or sacral region. The abnormal digital appendages involving the left heel and thigh in one boy are unique. Their presence plus the clefting and congenital skin defect of the skull suggested trisomy 13 syndrome; however, $\mathrm{G}$ banded chromosome analysis was normal. There is no evidence of any clefting or hearing loss in the parents nor is there any evidence to implicate a teratogen.

Winter and Donnai ${ }^{2}$ have drawn attention to the existence of a possible human homologue for the mouse mutant disorganisation (DS) ${ }^{3}$ which is located on mouse chromosome $14 .^{4}$ An important feature of this mutant is the fact that it can cause anomalies in many, apparently unrelated, systems and can act over a long period of time in organogenesis. There is a great variability in the anomalies that can be caused by this mutant and those which we have described in our first patient are reasonably consistent with such a possibility. He showed facial and palatal clefting, aberrant digital formation in unusual places, sacral lipoma, and a small skin defect of the scalp. His brother has fewer defects to suspect the DS mutant, but those that he has are consistent with the possibility. A problem such as sensorineural deafness has not been assessed in mice with the DS mutant. In the mouse this mutant is the result of a semidominant gene which is lethal in homozygotes and has variable penetrance in heterozygotes. In the family reported here, the evidence is more suggestive of autosomal recessive or $\mathrm{X}$ linked recessive inheritance. However, other explanations are certainly possible, for example, non-penetrance of the gene or germinal mosaicism in one parent.

We thank the late Dr J W Whitelaw who made the original referral of this family and many subsequent physicians for their cooperation and reports, including Drs J R MacLean, R H Marshall, H M Bell, J M Stephenson, A D Courtemanche, P K Will, and C D McLean. We would particularly like to thank the parents and the patients for their help and excellent cooperation. We thank Marie Bruce for secretarial assistance. Financial support from the Alberta Children's Hospital Foundation and the Medical Research Council of Canada Grant No 4539 is gratefully acknowledged.

1 Cohen MM. Syndromes with cleft lip and cleft palate. Cleft Palate F 1978;15:306-28.

2 Winter RM, Donnai D. A possible human homologue for the mouse mutant disorganisation. F Med Genet 1989;26:417-20.

3 Hummel KP. Developmental anomalies in mice resulting from action of the gene Disorganization, a semidominant lethal. Pediatrics 1959;23:212-21.

4 Lyon MF, Searle AG, eds. Genetic variants and strains of the laboratory mouse. 2nd ed. Oxford: Oxford University Press, 1989:92. 